\title{
Computational probing of temperature dependent unfolding of a small globular protein: From cold to heat denaturation
}

Changwon Yang ${ }^{1}$, Soonmin Jang ${ }^{1}$, and Youngshang Pak ${ }^{2 *}$

${ }^{1}$ Department of Chemistry, Sejong University, 209 Neungdong-ro, Gwangjin-gu, Seoul 05006, S. Korea

2 Department of Chemistry and Institute of functional materials, Pusan National University, Busandaehak-ro 63beon-gil, Geumjeong-gu, Busan 46241 S. Korea

*ypak@pusan.ac.kr

\section{Fine-tuning of all-atom protein force field}

Originally, we used the amber-fb 15 force field ${ }^{1}$ in conjunction with the tip3p-fb water model $^{2}$, because these ffs were more balanced versions of the standard amber 15 and tip $3 p$ models. In a preliminary benchmark test of the $1 \mathrm{DV} 0$ protein, the amber-fb15/tip3p-fb overestimated the thermal stability of this protein especially at the lower temperature region (see Figure S1). We found that this overestimation was largely due to too strong hydrophobic core of the UBA2 protein. To improve this overestimating profile, such strong hydrophobic core strength needs to be alleviated. For this purpose, we applied a previous NBFIX correction scheme ${ }^{3}$ for further tuning the amber-fb $15 \mathrm{ff}$. To modulate the hydrophobic core strength, the van der Waals ( $\mathrm{vdW}$ ) parameters $(\sigma$ and $\varepsilon$ ) of aliphatic (atom type CT) and aromatic (atom type CA) carbon atoms were adjusted to a unified set of $(\sigma, \varepsilon)$, so that only the pre-defined vdW pairs of CT/CT, CA/CA, and CA/CT are affected by these new parameters. Here, the $\sigma$ and $\varepsilon$ values of $\mathrm{CT}$ and CA were increased and decreased, respectively. For all other vdW pairs involving CT or CA, however, the original values of amber-fb15 are used (See Table S1 for changed parameters). This modification was helpful to properly weaken the strength of the hydrophobic core, leading to a muchimproved thermal denaturation profile (Figure S1).

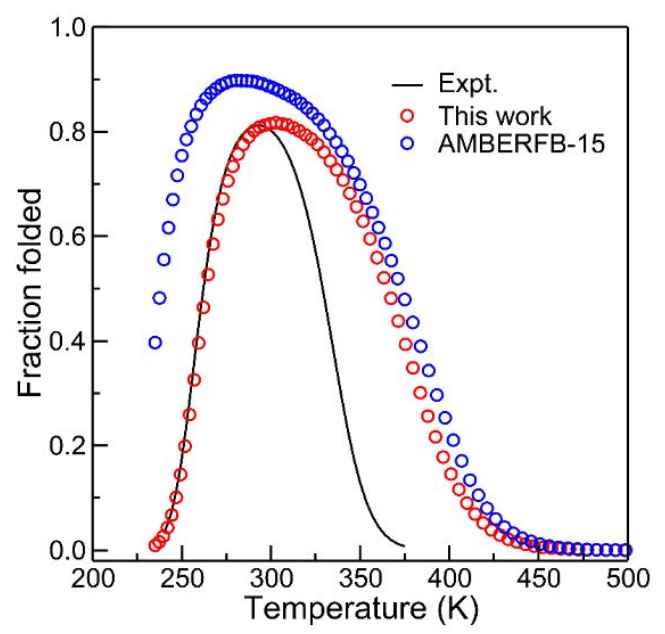

Figure S1. Simulated profile of folding fraction vs. temperature. The blue circle represents the preliminary result from $10 \mu$ s REMD using the original amber-fb15 in combination with tip $3 \mathrm{p}-\mathrm{fb}$. The red circle is the result from $17 \mu$ s REMD using the modified version of the amber-fb15 and the tip3p- 
fb. The black solid line is the experimental result. ${ }^{4}$

Table S1. Modified vdW parameter $(\sigma, \varepsilon)$ used in the current study.

\begin{tabular}{c|c|c|c}
\hline \hline \multirow{2}{*}{ Atom pair } & CT : CT & CT : CA & CA : CA \\
& $\sigma(\AA) / \varepsilon(\mathrm{kcal} / \mathrm{mol})$ & $\sigma(\AA) / \varepsilon(\mathrm{kcal} / \mathrm{mol})$ & $\sigma(\AA) / \varepsilon(\mathrm{kcal} / \mathrm{mol})$ \\
\hline \hline \multirow{2}{*}{ AMBER-FB15 } & $3.39967 / 0.1094(\mathrm{CT})$ & $3.39967 / 0.1094(\mathrm{CT})$ & $3.39967 / 0.0860(\mathrm{CA})$ \\
& $3.99967 / 0.0602(\mathrm{CT})$ & $3.99967 / 0.0602(\mathrm{CT}$ or $\mathrm{CA})$ & $3.99967 / 0.0602(\mathrm{CA})$ \\
\hline This work & \multirow{2}{*}{. } & $3.9967 / 0.0860(\mathrm{CA})$ & \\
\hline
\end{tabular}

\section{Convergence of the REMD simulation}

To show the convergence of the current simulation, the number of folded replicas out of a total 72 replicas in the REMD is monitored for a time period of $17 \mu$ s per replica. The fraction folded appears to reach a steady state after $6 \mu$ s (Figure S2A). Also, note that two simulated profiles of the folding fraction vs. temperature for each time block of the 7-12 and 12-17 $\mu$ s trajectories are well-overlapped (Figure S2B).

(A)

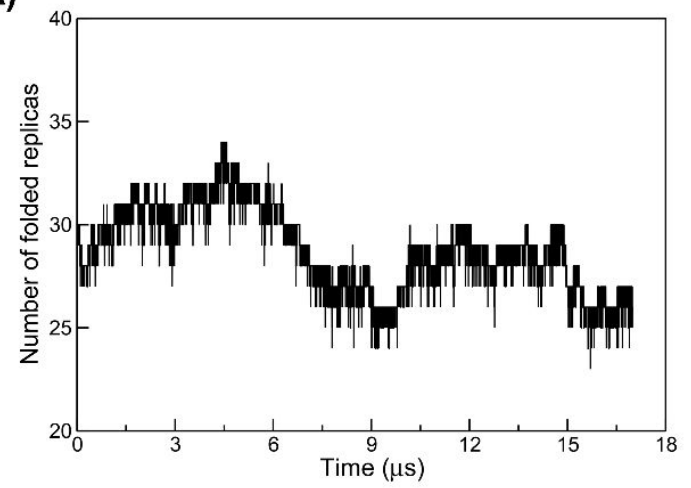

(B)

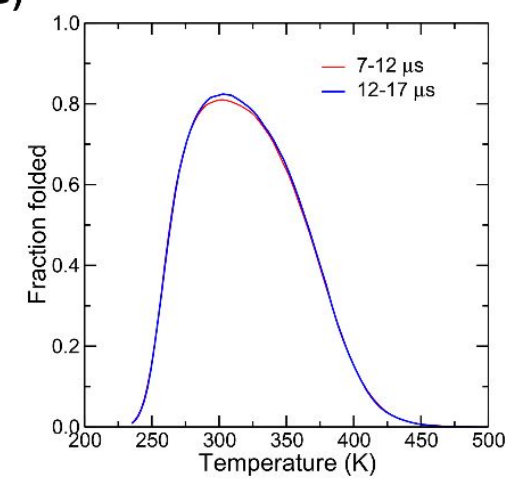

Figure S2. (A) Total number of folded replicas vs. time from $17 \mu$ s REMD simulation at the NPT condition. (B) Two overlapped profiles of folding fraction vs. temperature obtained from two time blocks of 7-12 and 12-17 $\mu$ s trajectories (the initial $7 \mu$ s trajectory is discarded for the analysis).

\section{Thermodynamic parameters determined from the two-state model}

The fold and unfold states are well-defined by a native contact $Q .{ }^{5}$ Here a folding criterion of $Q>0.7$ is used. The native contact parameter $Q$ is defined as

$$
Q=\frac{1}{N} \sum_{i<j} \frac{1}{\left(1+\exp \left(\beta\left(r_{i j}-\lambda r_{i j}^{0}\right)\right)\right)}
$$


Total $N$ pairwise summation is run over all heavy atoms satisfying the following conditions:

1. Residue number differences are more than 3.

2. The distance between heavy atoms $i$ and $j\left(r_{i j}{ }^{0}\right)$ is less than $4.5 \AA$ in the native state.

3. The smoothing parameter $\beta$ was chosen to $5 \AA^{-1}$ and the fluctuation parameter $\lambda$ was set to 1.5 .

This choice nicely separates the fold and unfold basins in the free energy surface over a broad temperature range. From the REMD structural ensembles, the profile of folding fraction $f$ vs. $T$ was obtained. Then, based on a two-state folding model, $f(T), \Delta H(T)$, and $\Delta S(T)$ can be expressed as

$$
\begin{aligned}
& f(T)=\frac{1}{(1+\exp (-(\Delta H-T \Delta S) / R T)} \\
& \Delta H(T)=\Delta H_{298 K}+\int_{298 K}^{T} \Delta C_{p}\left(T^{\prime}\right) d T^{\prime} \\
& \Delta S(T)=\Delta S_{298 K}+\int_{298 K}^{T} \Delta C_{p}\left(T^{\prime}\right) / T^{\prime} d T^{\prime} \\
& \Delta C_{p}(T)=\alpha T^{-1}+\beta+\gamma T
\end{aligned}
$$

The simulated data points of $f$ vs. $T$ are fit to Eq. (S2) to determine $\alpha, \beta$, and $\gamma$ values in the heat capacity function $\Delta C_{p}(T), \Delta H_{298 K}$, and $\Delta S_{298 K}$ (See Table S2 and Figure S3).

Table S2. Thermodynamics parameters on unfolding

\begin{tabular}{ccccc}
\hline \hline $\begin{array}{c}\Delta H_{298 K} \\
\left(\mathrm{kcal} \mathrm{mol}^{-1}\right)\end{array}$ & $\begin{array}{c}\Delta S_{298 K} \\
\left(\mathrm{cal} \mathrm{mol}^{-1} \mathrm{~K}^{-1}\right)\end{array}$ & $\begin{array}{c}\alpha \\
\left(\mathrm{kcal} \mathrm{mol}^{-1}\right)\end{array}$ & $\begin{array}{c}\beta \\
\left(\mathrm{kcal} \mathrm{mol}^{-1} \mathrm{~K}^{-1}\right)\end{array}$ & $\begin{array}{c}\gamma \\
\left(\mathrm{kcal} \mathrm{mol}^{-1} \mathrm{~K}^{-2}\right)\end{array}$ \\
\hline \hline-1.29274 & -7.27314 & 1425.2 & -8.15401 & 0.0121306 \\
\hline
\end{tabular}

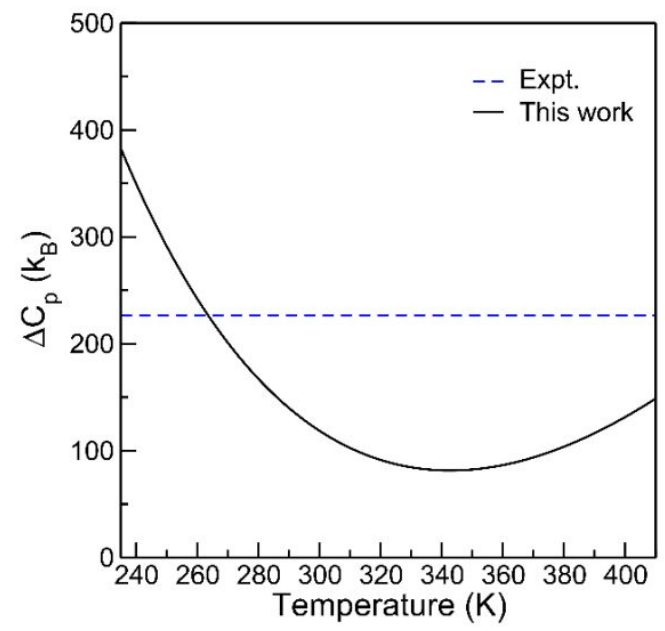


Figure S3. Temperature dependence of the heat capacity change upon unfolding with the fitting parameters $\alpha, \beta$, and $\gamma$ obtained from a two-state fit. The blue broken-line is the experimental value resulting from assuming a constant $\Delta C_{p \cdot}{ }^{4}$

\section{Conformational entropy estimation using the Gaussian mixture (GM) method}

In the Gaussian mixture (GM) entropy calculation method, ${ }^{6}$ a probability distribution in the torsional angle space $(\boldsymbol{q})$ is assumed to be a linear combination of $k$ multivariate Gaussian functions, such that $p_{k}$ $(\boldsymbol{q})=\sum_{i=1}^{k} w_{i} N\left(\boldsymbol{q} ; \boldsymbol{\mu}_{\boldsymbol{i}}, \boldsymbol{\sigma}_{\boldsymbol{i}}\right)$, where $w_{i}$ is a weighting factor and $N\left(\boldsymbol{q} ; \boldsymbol{\mu}_{\boldsymbol{i}}, \boldsymbol{\sigma}_{\boldsymbol{i}}\right)$ is the Gaussian distribution function in the torsional angle space $\boldsymbol{q}$ with mean $\boldsymbol{\mu}_{\boldsymbol{i}}$ and covariance matrix $\boldsymbol{\sigma}_{\boldsymbol{i}}$. In the above probability distribution, the number of Gaussian component $k$, mean $\boldsymbol{\mu}_{\boldsymbol{i}}$, weighting factor $w_{i}$ and covariance matrix $\sigma_{i}$ are unknown variables to be determined. These unknown variables are obtained by a greedy expectation-maximization (EM) method proposed by Verbeek et al. ${ }^{7}$ From a given wellconverged conformational ensemble $\boldsymbol{Q}=\left(\boldsymbol{q}_{\mathbf{1}}, \ldots, \boldsymbol{q}_{\boldsymbol{n}}\right)$ of sample size $n$, the information entropy $H$ is related to a maximized log-likelihood divided by the sample size $n$. This maximized log-likelihood $L$ can be obtained directly from this EM procedure:

$$
H=-\frac{L\left(\boldsymbol{Q}, p_{k}\right)}{n}=-\frac{1}{n} \sum_{i=1}^{n} \log p_{k}\left(\boldsymbol{q}_{\boldsymbol{i}}\right)
$$

Then, the conformational entropy is $S=R H$, where $R$ is the gas constant. The Gaussian mixture (GM) method for conformational entropy calculations requires a training set $\boldsymbol{Q}$ of protein conformation ensembles. ${ }^{6}$ As a result, protein conformational entropy changes upon unfolding $\Delta S_{c}=S_{c, D}-S_{c, N}$ depend on the data size. To test the size-dependence, $\Delta S_{c}$ was plotted in terms of data size. As shown in Figure S4, the calculated $\Delta S_{c}$ values well converge with the data size of 400,000 (400k) for low, medium, and high temperatures.

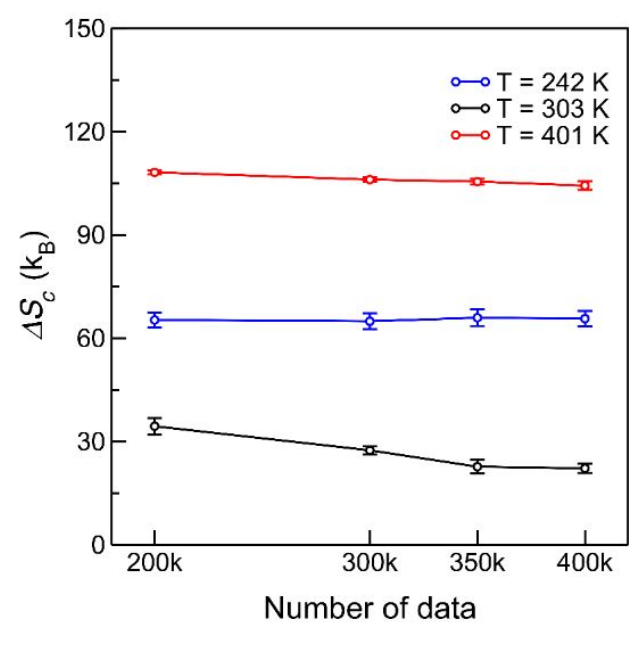


Figure S4. Sample size dependence of conformational entropy difference upon unfolding $\left(\Delta S_{c}\right)$ in the unit of Boltzmann's constant $\left(k_{B}\right)$. The entropy calculation was repeated 5 times for both fold and unfold states at each temperature and the mean and standard deviation were obtained.

\section{Comparison of representative denatured structures at various temperatures}

Hierarchical cluster analysis proposed by Daura et al ${ }^{8}$ are carried out to find representative unfold structures on the simulated free energy surfaces at $T_{\text {low }}=235 \mathrm{~K}, T_{\max } \approx 306 \mathrm{~K}$, and $T_{\text {high }}=452 \mathrm{~K}$. In the cluster analysis, a total of 100,000 structures from the REMD trajectory at each temperature were used with a C $\alpha$-RMSD cut-off $3.0 \AA$. Representative structures corresponding to unfold state free energy basins at the three temperatures are given in Figure S5. 

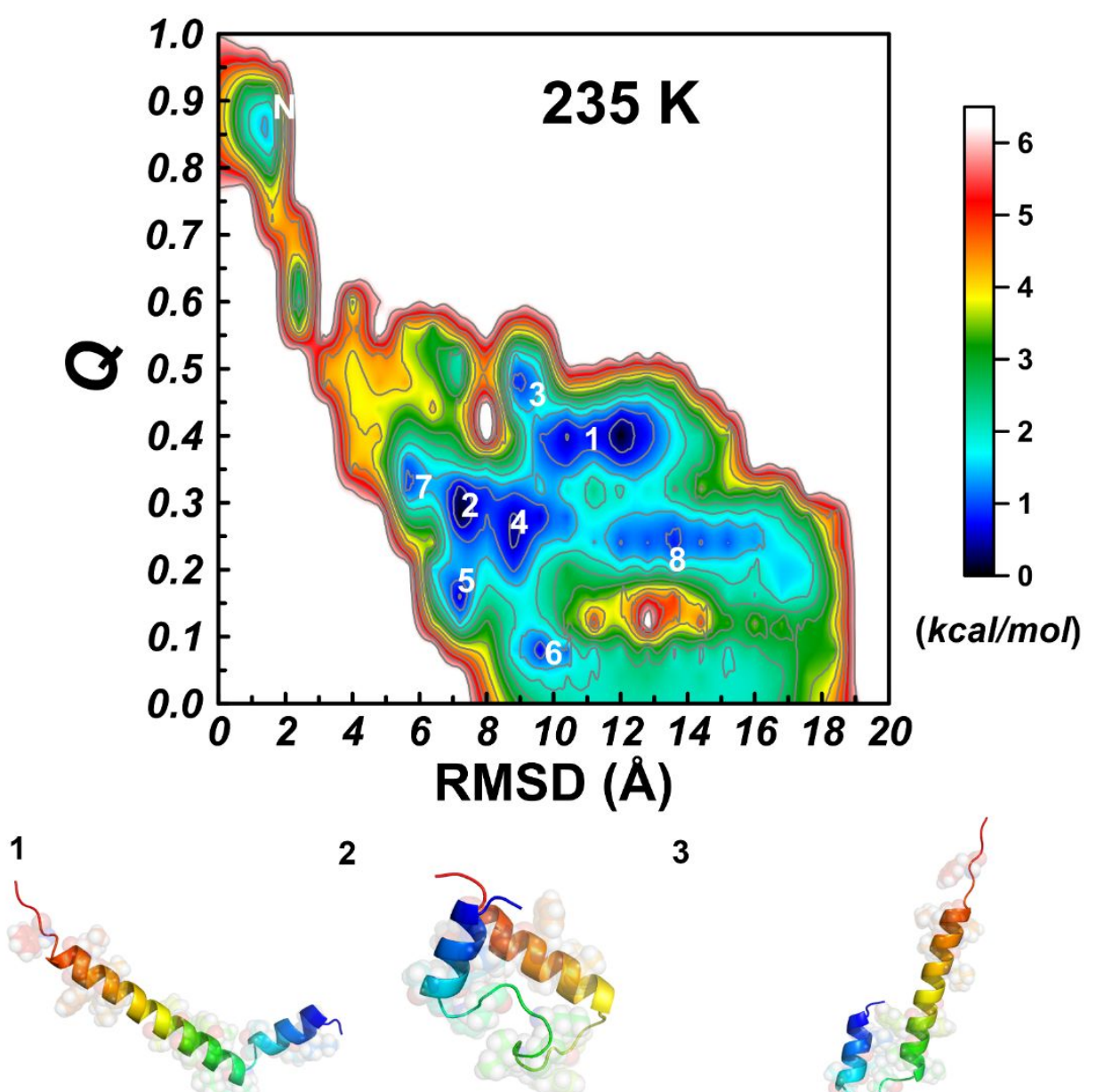

2

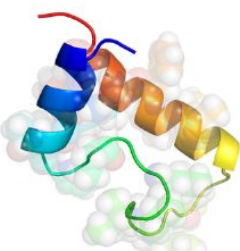

3

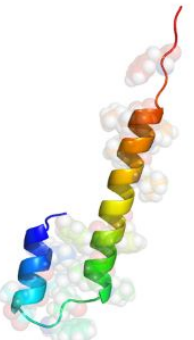

4

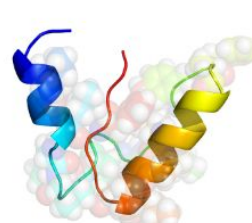

7

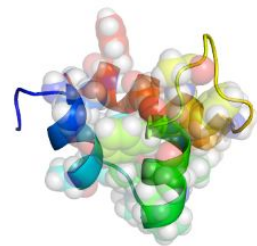

5

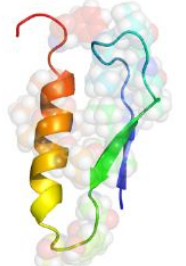

8
6

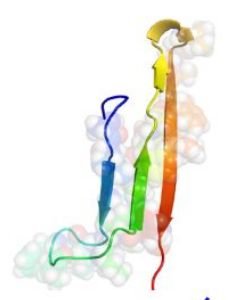

$\mathbf{N}$

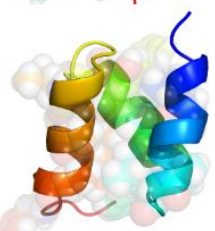

Figure S5 (A). Denatured structures at $235 \mathrm{~K}$. Each denatured structure corresponding to the eight unfolded free energy basins labeled from 1 to 8 is taken from the largest cluster population on each basin. Non-polar side chains are drawn by a transparent space-filling model. The native structure of the $\mathrm{UBA}(2)$ domain is denoted as $\mathrm{N}$. 


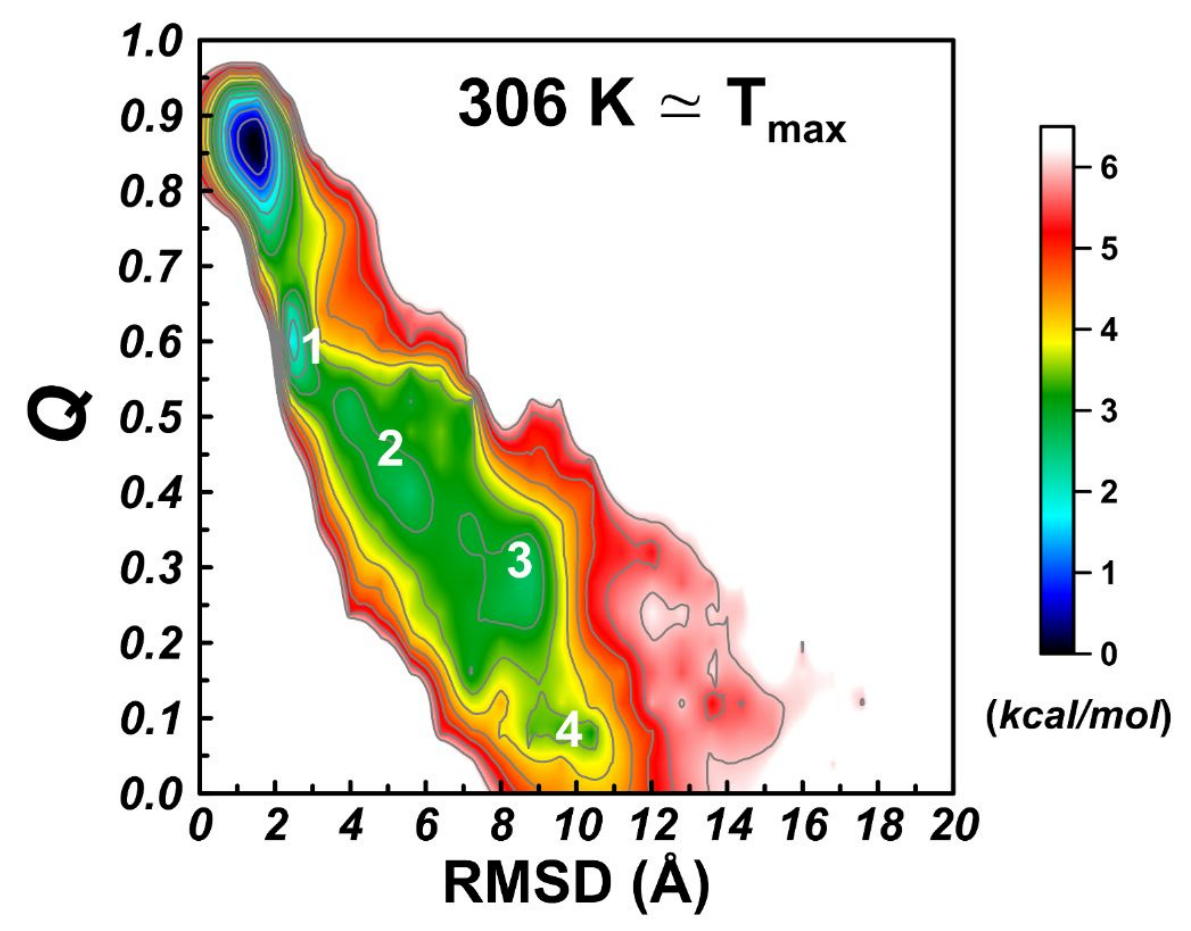

1

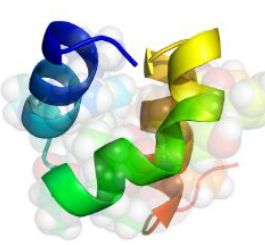

3

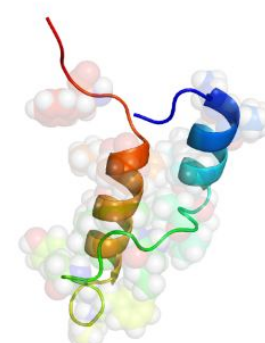

2

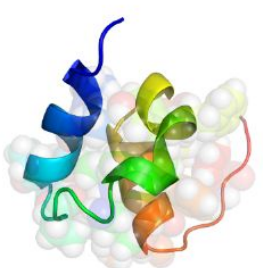

4

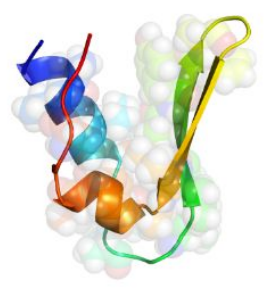

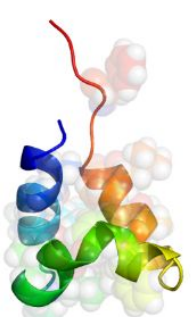
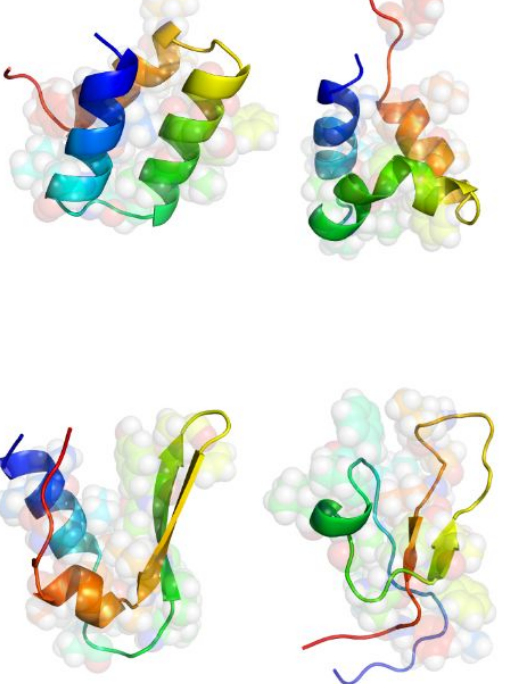

Figure S5 (B). Representative denatured structures at $306 \mathrm{~K}$. A pair of denatured structures corresponding to the four unfolded free energy basins labeled from 1 to 4 are taken from the largest and second-largest cluster populations on each basin. 

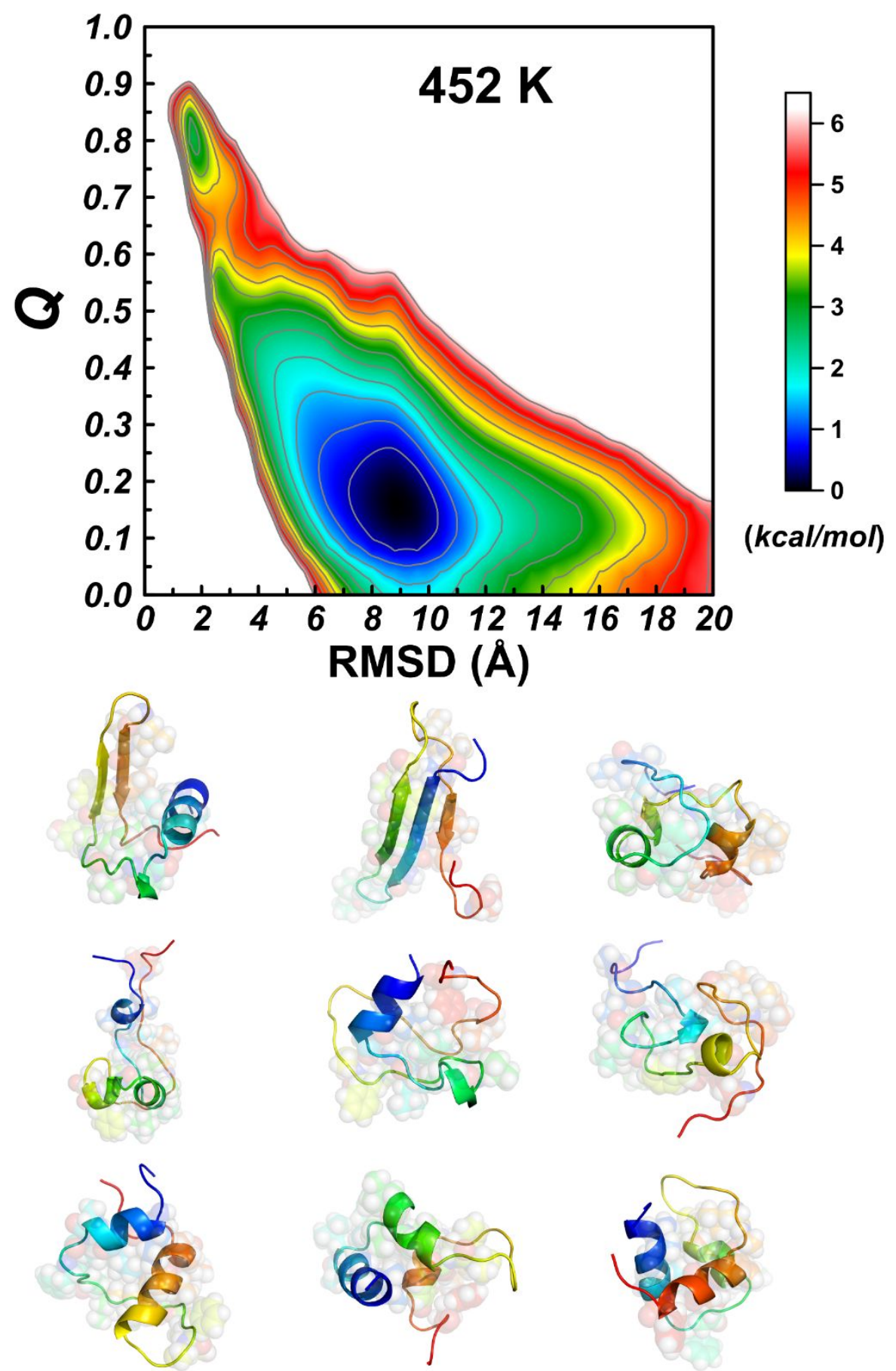

Figure S5 (C). Representative denatured structures at $452 \mathrm{~K}$. Denatured structures are chosen from the 9 largest cluster populations at a single large unfold free energy basin $(0.08<Q<0.25,7<$ RMSD $<10.5 \AA)$. 


\section{References}

(1) Wang, L. P.; McKiernan, K. A.; Gomes, J.; Beauchamp, K. A.; Head-Gordon, T.; Rice, J. E.; Swope, W. C.; Martinez, T. J.; Pande, V. S., Building a More Predictive Protein Force Field: A Systematic and Reproducible Route to AMBER-FB15. J. Phys. Chem. B 2017, 121, 4023-4039.

(2) Wang, L. P.; Martinez, T. J.; Pande, V. S., Building Force Fields: An Automatic, Systematic, and Reproducible Approach. J. Phys. Chem. Lett. 2014, 5, 1885-1891.

(3) Yoo, J.; Aksimentiev, A., Refined parameterization of nonbonded interactions improves conformational sampling and kinetics of protein folding simulations. The journal of physical chemistry letters 2016, 7, 3812-3818.

(4) Buchner, G. S.; Shih, N.; Reece, A. E.; Niebling, S.; Kubelka, J., Unusual Cold Denaturation of a Small Protein Domain. Biochemistry 2012, 51, 6496-6498.

(5) Best, R. B.; Hummer, G.; Eaton, W. A., Native contacts determine protein folding mechanisms in atomistic simulations. Proc. Natl. Acad. Sci. U. S. A. 2013, 110, 17874-17879.

(6) Gyimesi, G.; Zavodszky, P.; Szilagyi, A., Calculation of Configurational Entropy Differences from Conformational Ensembles Using Gaussian Mixtures. J. Chem. Theory Comput. 2017, 13, 29-41.

(7) Verbeek, J. J.; Vlassis, N.; Krose, B., Efficient greedy learning of Gaussian mixture models. Neural Comput. 2003, 15, 469-485.

(8) Daura, X.; Gademann, K.; Jaun, B.; Seebach, D.; Van Gunsteren, W. F.; Mark, A. E., Peptide folding: when simulation meets experiment. Angew. Chem. Int. Ed. 1999, 38, 236-240. 